\title{
Origin of Low Energy $d-d$ Excitations Observed on Wet Chemically Prepared Cobalt Bearing Nanoparticles by 2p3d Resonant X-ray Emission Spectroscopy
}

\author{
Matti M. van Schooneveld, ${ }^{*}{ }^{\dagger}$ Amélie Juhin, ${ }^{\dagger}$ Carlos Campos-Cuerva, ${ }^{\dagger}$ Thorsten Schmitt, ${ }^{\ddagger}$ \\ and Frank M. F. de Groot* ${ }^{*}$ \\ ${ }^{\dagger}$ Inorganic Chemistry and Catalysis, Debye Institute for Nanomaterials Science, Utrecht University, Universiteitsweg 99,3584 CG \\ Utrecht, The Netherlands \\ ${ }^{\ddagger}$ Swiss Light Source, Paul Scherrer Institut (PSI), CH-5232 Villigen PSI, Switzerland
}

Supporting Information

\begin{abstract}
The 2p3d resonant X-ray emission spectroscopic (RXES) measurements on 8.4 and $5.0 \mathrm{~nm}$ cobalt and $3.6 \mathrm{~nm}$ cobalt-nickel nanoparticles coated with oleate molecules are provided. The spectra reveal low energy resonant Raman features at 0.3 and $0.75 \mathrm{eV}$. In combination with time-dependent density functional theory (TD-DFT) and ligand field multiplet (LFM) calculations, these are ascribed to $d-d$ excitations of cobalt ions in a low symmetry ligand field. Two different chemical environments of the ion may cause the transitions. In the first model cobalt ions in the nanoparticle outer atomic layer, resulting from the adsorbate binding, cause the excitations. These are transitions from a mixture of ${ }^{4} \mathrm{~B}_{1 \mathrm{~g}}$ plus ${ }^{4} \mathrm{E}_{\mathrm{g}}$ to ${ }^{4} \mathrm{E}_{\mathrm{g}}$ at $0.3 \mathrm{eV}$. At $0.75 \mathrm{eV}$ transitions to ${ }^{4} \mathrm{~B}_{2 \mathrm{~g}}$ and ${ }^{4} \mathrm{~A}_{1 \mathrm{~g}}$ take place. In the alternative model the excitations occur in a cobalt molecular species that might coexist with the nanoparticles. Here the transitions are from ${ }^{4} \mathrm{~A}_{2 g}$ to ${ }^{4} \mathrm{E}_{\mathrm{g}}$ and to ${ }^{4} \mathrm{E}_{\mathrm{g}}$ plus ${ }^{4} \mathrm{~B}_{2 \mathrm{~g}}$ at 0.3 and $0.75 \mathrm{eV}$, respectively. On the basis of two-dimensional 2p3d RXES planes of the models and the differences between the three different nanoparticle systems, we exclude the first model and conclude that the metallic particles indeed coexist with varying minor degrees of molecular species. We observe however a second type of cobalt species, which is possibly related to surface-ligated cobalt ions.

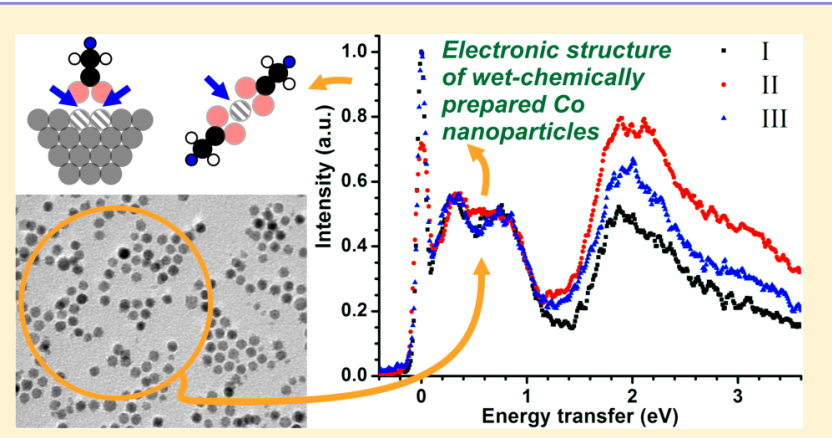

\section{INTRODUCTION}

Direct measurement of the electronic structure of chemical bonds formed at a surface is far from trivial but is important. ${ }^{1,2}$ In catalysis the electron configuration and dynamics within the first atomic layers control to a large extent how molecules adsorb, form bonds, and eventually desorb. ${ }^{3}$ Great progress in understanding such processes was made by the application of sensitive surface science techniques ${ }^{4,5}$ such as scanning tunneling microscopy (STM). ${ }^{6}$ Despite this, it remains a challenge to specifically probe the bond between a surface atom and the atom within a molecule that binds with the surface. ${ }^{7}$ As an analogy, little is known about the bond orbitals between nanoparticles and surfactants (although many studies improved our understanding of nanoparticle synthesis, size, morphology, crystal structure, and elemental composition).

Considering the "surface-molecule bond challenge", the recent developments in resonant X-ray emission spectroscopy $(\mathrm{RXES})^{8-12}$ are interesting. In RXES (also known as resonant inelastic X-ray scattering, RIXS) the studied material is irradiated with X-rays to excite a core electron to empty electronic states. The term "resonant" implies excitation at a material-specific core electron binding energy, in contrast to normal or "nonresonant" X-ray emission where photon 
to depths that depend on the photon attenuation length, which is up to $200 \mathrm{~nm}$ at the cobalt $2 \mathrm{p}$ resonance. ${ }^{16}$ We have recently reported however that, on a metallic substrate, ${ }^{17} \mathrm{~d}-\mathrm{d}$ and other resonant X-ray Raman features are nonobservable, and that this can be used to specifically probe $d-d$ excitations related to metal ion surface species. On cobalt nanoparticles we showed that surface oxidation of more than the first atomic layer is revealed through $\mathrm{d}-\mathrm{d}$ excitations characteristic for a highly symmetric octahedral ligand field. ${ }^{18}$ Here we report extensive 2p3d RXES measurements on 8.4 and $5.0 \mathrm{~nm}$ metallic cobalt and $3.6 \mathrm{~nm}$ metallic cobalt-nickel nanoparticles, coated with oleates. We show that 2 p3d RXES at several excitation energies reveals a minority of molecular species to coexist with metallic nanoparticles, and we discuss to what extent the technique is sensitive to surface-adsorbate bonds.

\section{EXPERIMENTAL SECTION}

Experimental and computational details are given in the Supporting Information. All nanoparticle syntheses and characterizations were performed under exclusion of air. Briefly, the $\varepsilon$-Co particles were synthesized through the Puntes method ${ }^{19}$ and the fcc $\mathrm{Co}_{0.65} \mathrm{Ni}_{0.35}$ particles were synthesized through a cobalt carbonyl acetonation scheme. ${ }^{20}$ The nanocrystals were washed and characterized by transmission electron microscopy (TEM), energy dispersive X-ray spectroscopy (EDX), and X-ray powder diffraction (XRD) before synchrotron X-ray measurements were performed.

For $2 \mathrm{p}$ XAS and $2 \mathrm{p} 3 \mathrm{~d}$ RXES the nanoparticles were dropcasted on deoxygenated $\mathrm{Si}$ wafers. ${ }^{21,22}$ Total electron yield (TEY) XAS spectra were acquired by measurement of the drain current and sampled with 25 points $\mathrm{eV}^{-1}$. The RXES spectra were measured using the high-resolution Super Advanced X-ray Emission Spectrometer (SAXES) at the ADvanced RESonant Spectroscopies (ADRESS; X03MA) beamline of the SLS. ${ }^{13,14}$ A scattering geometry was used in which the angle between the incoming light wave vector and the outgoing one was $90^{\circ}$. The incoming light was polarized linear parallel (depolarized geometry or linear horizontal, LH) to the scattering plane with a grazing incident angle of $20^{\circ}$. Measuring at grazing incidence minimizes self-absorption of the elastic peak. Measuring at LH polarization suppresses the cross section of the elastic peak with respect to the resonant features. ${ }^{23,24}$ By positioning the beam at a non-cobalt amorphous reference and varying the incoming energy from 770 to $780 \mathrm{eV}$ with $0.5 \mathrm{eV}$ step $^{-1}$, the elastic peak was used to calibrate the RXES detector. The peaks were fitted with Gaussian functions, and the maxima of the fits were plotted against detector channels. The plot was fitted with a linear curve to determine the energy-channel response function. At the Co $2 \mathrm{p}_{3 / 2}$ XAS edge $(\sim 780 \mathrm{eV})$ the combined energy resolution of the RXES zero-loss peak was 88 $\mathrm{meV}$ full width at half-maximum (fwhm) for the nanoparticle data and $196 \mathrm{meV}$ fwhm for the cobalt oleate molecular complex. These resolutions were the best obtainable resolutions during the two different experiments at which the data were acquired. A similar calibration procedure was followed for the Ni 2p3d RXES spectra. The RXES spectra of the compounds were sampled with 52 points $\mathrm{eV}^{-1}$ and summed over 4-10 partial spectra that were acquired for $10 \mathrm{~min}$ each. To compare the spectra, they were normalized to the intensity of the maximum (Figure 2 ) or to the intensity of the $d-d$ excitations at 0.3 and $0.75 \mathrm{eV}$ (Figures 3a, 4c, 5, and 7). Spectra were not normalized to the elastic line, because many parameters influence its intensity including sample surface roughness and experimental resolution. The pressure in the analysis chamber was $\sim 10^{-8}$ mbar and the temperature was $20 \pm 5{ }^{\circ} \mathrm{C}$ during all measurements. For both XAS and RXES we did not notice any evolution of the spectra during the experiment.

\section{COMPUTATIONAL SECTION}

Density Functional Theory (DFT). Geometry optimization calculations were performed to determine the mode of strongest ligand bonding on a cobalt surface. ${ }^{25-31}$ For the calculations we used the ORCA software package. ${ }^{25,26}$

Time-Dependent DFT (TD-DFT). After we identified ground state configurations of complexes, TD-DFT ${ }^{32}$ was used to calculate part of the optical transitions in the $0-2.5 \mathrm{eV}$ regime for comparison with RXES spectra. Time-dependent theory is based on the Runge-Gross theorem, ${ }^{33}$ the timedependent analogue of the Hohenberg-Kohn-Sham formalisms for the description of the ground state electron density. ${ }^{25,34}$ Note that for this study only excitations of $\leq 1$ $\mathrm{eV}$ were used for comparison with the RXES spectra since excitations to higher lying states are frequently of Rydberg or double excitation character for which TD-DFT has been reported to fail. ${ }^{32,35,36}$

Ligand Field Multiplets (LFM). ${ }^{37}$ The $2 \mathrm{p}$ XAS and $2 \mathrm{p} 3 \mathrm{~d}$ RXES spectra were simulated using the multiplet program Charge Transfer Multiplet for XAS (CTM4XAS). ${ }^{38-41}$ Only ligand field effects were included, since charge transfer (CT) effects will only a little modify the $2 p$ XAS and 2 p3d RXES spectra of $\mathrm{Co}^{\mathrm{II}}$ carboxylates, except for some additional intensity at 4-5 eV in the RXES spectra acquired at excitation energy $e .{ }^{15}$ This can be seen by comparing spectra measured at energy $e$ with those acquired at $a-d$. We ascribe the minor effect of CT on the 2p3d RXES spectra to the localized nature of the $2 \mathrm{p}$ to $3 \mathrm{~d}$ excitation, plus, for $\mathrm{Co}^{\mathrm{II}}$ carboxylate types, the relatively small extent of $3 \mathrm{~d}$-electron delocalization on the oxygen atoms. ${ }^{15}$ The electronic structure parameter values used in the spectral interpretation are given in Table 1 . We

Table 1. Energetic Values (in eV) of the Ligand Field Multiplet Parameters Used in Model A/Model B

\begin{tabular}{llll} 
& \multicolumn{1}{c}{ initial state } & intermediate state & \multicolumn{1}{c}{ final state } \\
$\mathrm{Co}^{2+}$ config & $2 \mathrm{p}^{6} 3 \mathrm{~d}^{7}$ & $2 \mathrm{p}^{5} 3 \mathrm{~d}^{8}$ & $2 \mathrm{p}^{6} 3 \mathrm{~d}^{8} \mathrm{~d}$ \\
$\mathrm{~F}^{2}{ }_{\mathrm{dd}}$ & $9.284 / 8.820$ & $9.917 / 9.421$ & $9.284 / 8.820$ \\
$\mathrm{~F}_{\mathrm{dd}}^{4}$ & $5.767 / 5.479$ & $6.166 / 5.858$ & $5.767 / 5.479$ \\
$\mathrm{~F}_{\mathrm{pd}}^{2}$ & - & $5.808 / 5.518$ & - \\
$\mathrm{G}_{\mathrm{pd}}^{1}$ & - & $4.318 / 4.102$ & - \\
$\mathrm{G}_{\mathrm{pd}}^{3}$ & - & $2.455 / 2.333$ & - \\
$\zeta_{3 \mathrm{~d}}$ & $0.033 / 0.050$ & $0.042 / 0.062$ & $0.033 / 0.050$ \\
$\zeta_{2 \mathrm{p}}$ & - & $9.748 / 9.748$ & - \\
$10 \mathrm{Dq}$ & $-0.200 / 0.600$ & $-0.200 / 0.600$ & $-0.200 / 0.600$ \\
$\mathrm{Ds}$ & $-0.050 / 0.150$ & $-0.050 / 0.150$ & $-0.050 / 0.150$ \\
$\mathrm{Dt}$ & $-0.070 / 0.000$ & $-0.070 / 0.000$ & $-0.070 / 0.000$ \\
$\Gamma($ fwhm $)$ & - & $0.400 / 0.400$ & $0.020 / 0.020$ \\
$G(\mathrm{fwhm})$ & - & $0.200 / 0.200$ & $0.100 / 0.100$ \\
\hline
\end{tabular}

approximate cobalt to have a formal $\mathrm{Co}^{\mathrm{II}}$ valence in models $A$ and $B$ (see Results for an explanation of the models). Co ${ }^{\text {II }}$ can be described effectively by a $2 \mathrm{p}^{6} 3 \mathrm{~d}^{7}$ ground state configuration. For the $2 p$ XAS spectra all electric dipoleallowed electron transitions to a $2 \mathrm{p}^{5} 3 \mathrm{~d}^{8}$ intermediate state are calculated, together with their oscillator strengths. The $\mathrm{Co}^{\mathrm{II}}$ $2 \mathrm{p} 3 \mathrm{~d}$ RXES spectra are simulated using $2 \mathrm{p}^{6} 3 \mathrm{~d}^{7} \rightarrow 2 \mathrm{p}^{5} 3 \mathrm{~d}^{8} \rightarrow$ 
$2 \mathrm{p}^{6} 3 \mathrm{~d}^{8} \mathrm{~d}$ transitions as described by the resonant term of the Kramers-Heisenberg formula. ${ }^{42,43}$

Although the final state $2 \mathrm{p}^{6} 3 \mathrm{~d}^{8} \underline{\mathrm{d}}$ configuration is formally equal to $2 \mathrm{p}^{6} 3 \mathrm{~d}^{7}$, we use this notation to indicate that the final state may have an unstable hole in the $3 \mathrm{~d}$ states.

The radial parts of the Slater integrals describing direct Coulomb and Coulomb exchange interaction of the cobalt ions $\left(F_{\mathrm{dd}}^{2}, \mathrm{~F}_{\mathrm{dd}}^{4}, \mathrm{~F}_{\mathrm{pd}}^{2}, \mathrm{G}_{\mathrm{pd}}^{1}\right.$ and $\left.\mathrm{G}_{\mathrm{pd}}^{3}\right)$ were not scaled in model $\mathrm{A}$, and all scaled to $95 \%$ of the free ion values in model B (this corresponds to 80 and $76 \%$ of the HF values). Reduction of the Slater integrals is done to effectively describe $3 \mathrm{~d}$ electron delocalization on ligands (the nephelauxetic effect ${ }^{44}$ ). The $3 \mathrm{~d}$ spin-orbit coupling, $\zeta_{3 \mathrm{~d}}$, was reduced here to 50 and $75 \%$ of its atomic $\mathrm{HF}$ value in models $\mathrm{A}$ and $\mathrm{B}$, respectively. The reason was previously discussed for $\mathrm{Co}^{\mathrm{II}}$ in $\mathrm{CoO}^{24}$ For effective descriptions of the ligand field the $D_{4 h}$ point group symmetry is used to simulate the effects of the carboxylate ligands with parameters $10 \mathrm{Dq}$, Ds, and Dt. ${ }^{10}$ The XAS transitions are finally convoluted with a Lorentzian function $\Gamma 2 \mathrm{p}_{3 / 2}$ to account for the $2 p_{3 / 2}$ core hole lifetime broadening at the $2 p_{3 / 2}$ XAS edge. A second convolution is done with a Gaussian function $G$ (fwhm) to account for instrumental broadening. All XAS spectra were calculated at $17{ }^{\circ} \mathrm{C}(290 \mathrm{~K})$. The RXES spectra are convoluted with Lorentzians accounting for the intermediate $\left(\Gamma 2 \mathrm{p}_{3 / 2}\right)$ and final $(\Gamma 3 \mathrm{~d})$ state lifetimes of the hole. Gaussians accounting for monochromator- and spectrometerinduced broadening were applied to convolute the spectra further. RXES calculations took the interference effects between $\mathrm{X}$-ray absorption and emission into account and were all done for the ground state, which formally relates to a situation at $-273.15{ }^{\circ} \mathrm{C}(0 \mathrm{~K})$. In the Supporting Information, Figure S4, the Boltzmann-distributed population of excited states at $17^{\circ} \mathrm{C}$ $(290 \mathrm{~K})$ is taken into account for model A. Although excited states are accessible at room temperature, these do not significantly affect the spectra, and the same was shown for model B previously. ${ }^{15}$ The spectra were calculated with the transition probabilities that correspond to the experimental $\mathrm{LH}$ polarization.

From TD-DFT to LFM. For model A in Figure 4, the origin and energies of the transitions as calculated by TD-DFT are used as restrictions for the LFM calculations. For this purpose the LFM multiplets, being many-electron states, were described as the sum of single particle states using an orbital projection method. ${ }^{45,46}$ The $3 \mathrm{~d}^{n}$ states were described in $D_{4 h}$ symmetry and decomposed into four different components $\left(a, b_{1}, b_{2}\right.$, and e), where $\alpha_{i}$ indicates the fraction of each orbital character:

$$
\Psi=\sum_{i ; k+l+m+n} \alpha_{i}\left|\mathrm{a}_{1}{ }^{k} \mathrm{~b}_{1}{ }^{l} \mathrm{~b}_{2}{ }^{m} \mathrm{e}^{n}\right\rangle
$$

Here $k+l+m+n$ are equal to three $3 \mathrm{~d}$ holes for the ground state $2 \mathrm{p}^{6} 3 \mathrm{~d}^{7}$ configuration. To determine the single particle fractions $\alpha_{i}$, the many state wave function of interest is projected onto single particle wave functions defined by combinations of empty $a_{1}, b_{1}, b_{2}$, and e holes. This is carried out by adding dummy $4 \mathrm{~s}$ orbitals and making a monopole transition from $3 d^{7} 4 s^{2} 4 s^{0}$ to $3 d^{7} 4 s^{1} 4 s^{1}$. The $4 s$ states are described without any correlations or interactions. The $3 \mathrm{~d}-3 \mathrm{~d}$ interactions and $3 \mathrm{~d}$ spin-orbit coupling of the final state $3 d^{7} 4 s^{1} 4 s^{1}$ configuration is set to zero, and the states are split purely by ligand field parameters. This provides transitions from the "correlated $3 \mathrm{~d}^{n}$ ground state" to "pure ligand field $3 \mathrm{~d}^{n}$ states", connecting these alternate descriptive bases, where the transition strengths yielding the $\alpha_{i}$ give the contributing configurations to the many state wave functions.

\section{RESULTS}

Figure 1a shows an artistic impression of the studied nanoparticles. They are depicted in the presence of

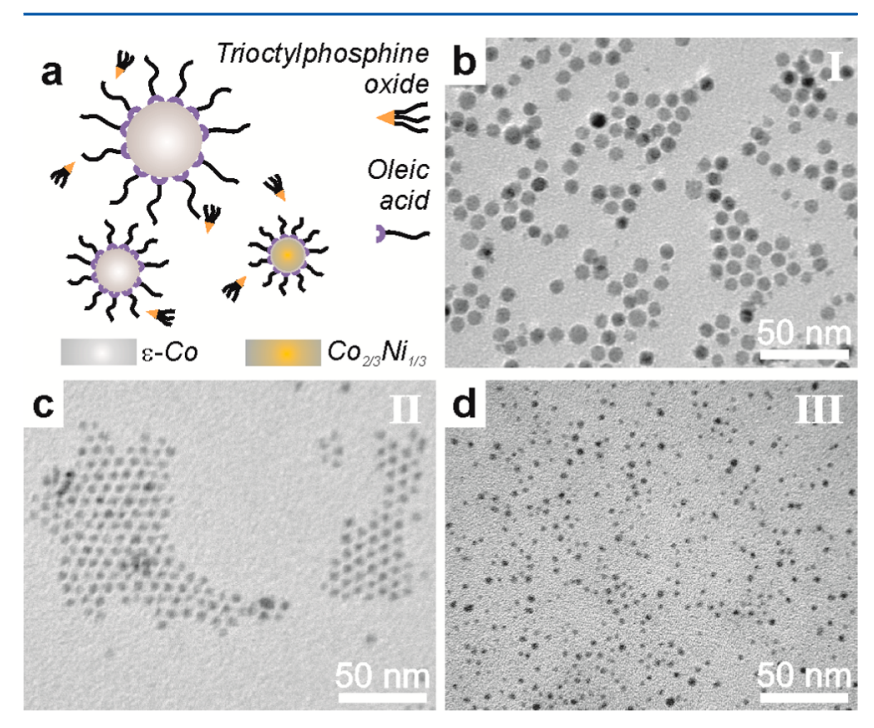

Figure 1. (a) Schematic representations and TEM images of the (b) $8.4 \mathrm{~nm} \varepsilon$-Co, (c) $5.0 \mathrm{~nm} \varepsilon$-Co, and (d) $3.6 \mathrm{~nm} \mathrm{fcc} \mathrm{Co}_{0.65} \mathrm{Ni}_{0.35}$ nanoparticles.

trioctylphosphine oxide (TOPO) and oleic acid (OA), which are used in their syntheses. TOPO acts as a kinetic growth controller, ${ }^{47}$ while OA attaches to the cobalt nanoparticle surface as the capping agent. ${ }^{48}$ Transmission electron microscopy (TEM) images of the $8.4 \mathrm{~nm} \varepsilon$-Co, $5.0 \mathrm{~nm} \varepsilon$-Co, and $3.6 \mathrm{~nm} \mathrm{fcc} \mathrm{Co}_{0.65} \mathrm{Ni}_{0.35}$ particles are shown in Figure $1 \mathrm{~b}-\mathrm{d}$. They are labeled systems I, II, and III throughout the text, respectively. The sizes refer to diameters of the spherical particles. Their respective polydispersities are 14, 14, and $21 \%$ (defined as the standard deviation over particle average diameter).

In Figure 2 the $2 \mathrm{p}_{3 / 2}$ XAS and 2p3d RXES spectra of systems I-III are shown. All Co XAS spectra show weak fine structure. This indicates that not all cobalt in the nanoparticle systems is metallic, nor that all is oxidized. An ensemble of cobalt metal atoms would exhibit a single $2 \mathrm{p}_{3 / 2}$ peak without fine structure, while only oxidized cobalt ions would show much stronger fine structure. ${ }^{24,49}$ This indicates the presence of either a single cobalt species with an electronic structure in between that of a metal and a metal oxide, which is impossible in the case of the chemically nonhomogeneous nanoparticle systems, or, more realistically, of multiple different cobalt species that combined give rise to the observed fine structure. The nickel $2 \mathrm{p}_{3 / 2}$ XAS spectrum in Figure $2 \mathrm{~d}$ shows a single white line implying all nickel to be metallic.

The corresponding 2p3d RXES spectra confirm the qualitative insights deduced from XAS. Five cobalt RXES spectra were acquired per nanoparticle batch at different excitation energies, $a-e$, as indicated in Figure 2. The five spectra are used to discriminate between spectral features that occur at a fixed transfer energy or a fixed emission energy. Transitions that are observed to occur at fixed transfer energy are transitions to excited states intrinsic to the material and 

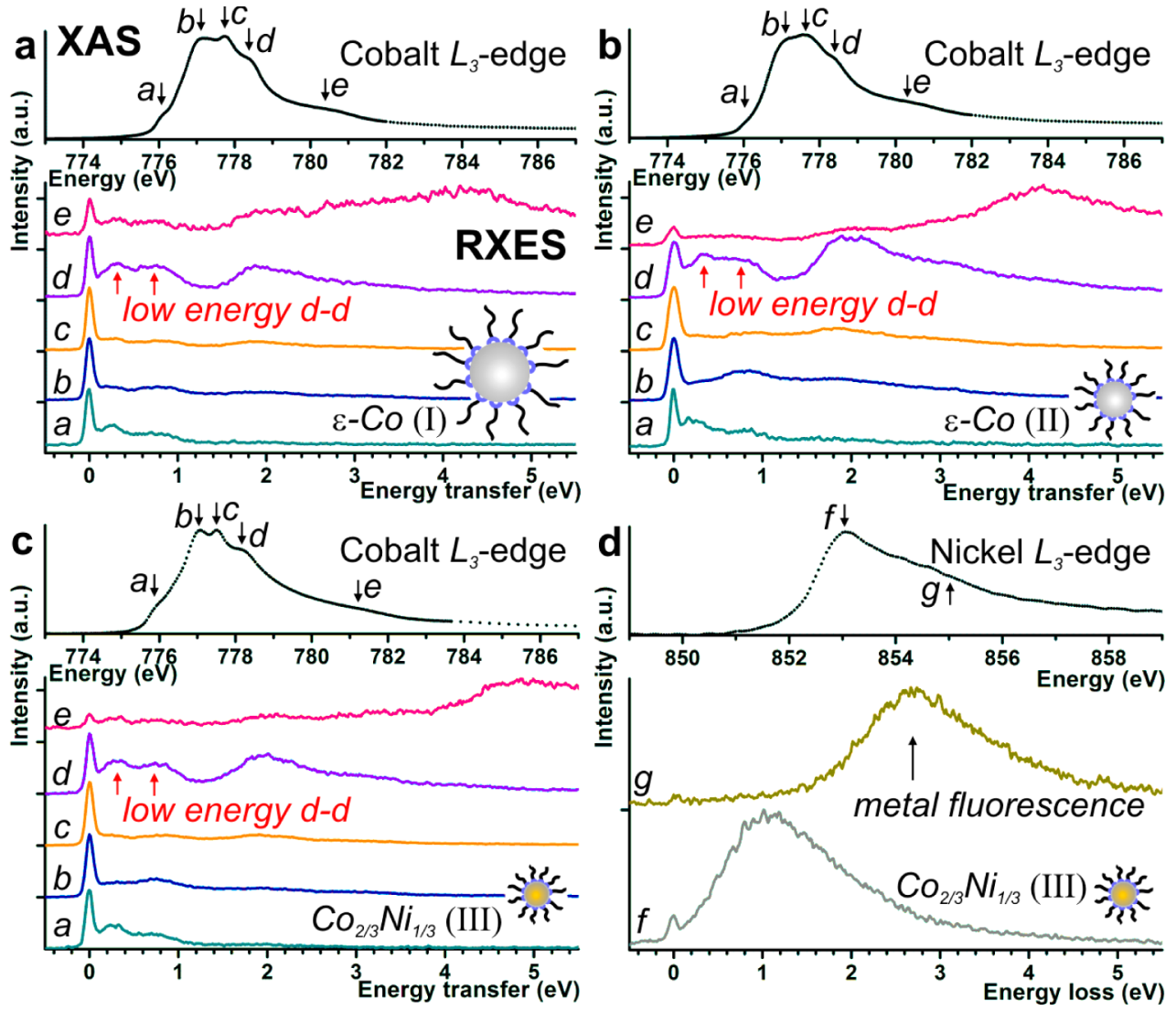

Figure 2. Experimental cobalt $2 \mathrm{p}_{3 / 2}$ XAS (top) and cobalt $2 \mathrm{p} 3 \mathrm{~d}$ RXES (bottom) spectra of the (a) $8.4 \mathrm{~nm} \varepsilon$-Co, (b) $5.0 \mathrm{~nm} \varepsilon$-Co, and (c) $3.6 \mathrm{~nm}$ fcc $\mathrm{Co}_{0.65} \mathrm{Ni}_{0.35}$ nanoparticles. (d) Similar spectra taken at the nickel edges on the $\mathrm{Co}_{0.65} \mathrm{Ni}_{0.35}$ particles. The RXES excitation energies are indicated in the XAS spectra with arrows and letters $a-e$ at the cobalt edge and $f$ and $g$ at the nickel edge.

require the same amount of energy irrespective of the excitation energy. These are called resonant Raman features. ${ }^{12,43}$ Peaks that occur at fixed emission energy originate from X-ray fluorescence.$^{50}$ It is observed three times in independent nanoparticle batches in the cobalt RXES spectra in Figure $2 \mathrm{a}-\mathrm{c}$ that two resonant Raman features at 0.3 and $0.75 \mathrm{eV}$ are evident. Resonant Raman features are also evident around 2 $\mathrm{eV}^{51}$ Cobalt nanoparticle oxidation to $\mathrm{CoO}$, as observed to happen for identical nanoparticles, cannot explain the observed peaks as this would yield no RXES peaks between 0.1 and $1 \mathrm{eV}$ and a strong peak around $1 \mathrm{eV}^{24}$ This means that virtually no cobalt species are present with 6-fold oxygen coordination as in CoO. The features do also not belong to metallic cobalt, because we experimentally observe metal spectra to be typically dominated by X-ray fluorescence only. ${ }^{18,52}$ The nickel $2 \mathrm{p} 3 \mathrm{~d}$ RXES spectra in Figure $2 \mathrm{~d}$ of the $\mathrm{Co}_{0.65} \mathrm{Ni}_{0.35}$ alloy nanoparticles demonstrate this: a weak elastic peak, X-ray fluorescence, and no resonant Raman features are observed. The spectra in Figure $2 \mathrm{~d}$ confirm the XAS assignment of the nickel being present as a formal metal. It is not surprising that the nickel is found to be metallic as (a) the cobalt oleate bond is stronger than that of nickel oleate, (b) cobalt is more readily oxidized than nickel, and (c) identical particles were characterized to consist of fcc cobalt-nickel alloys with likely an outer cobalt layer. ${ }^{20}$

The resonant Raman features at 0.3 and $0.75 \mathrm{eV}$, which we reproductively observed in the cobalt $2 \mathrm{p} 3 \mathrm{~d}$ RXES spectra as duplicated in Figure 3a, are features of considerable interest. Their energies are higher than typical phonon energies $(<0.2$ $\mathrm{eV}$ ) and are too low for typical $\mathrm{d}-\mathrm{d}(1-2 \mathrm{eV})$ or charge transfer $(>3 \mathrm{eV})$ excitations as seen in transition metal oxides. ${ }^{12}$ Moreover, they are unlikely to be collective magnetic excitations such as magnons since the peaks only appear in cobalt and not in nickel RXES of the $\mathrm{Co}_{0.65} \mathrm{Ni}_{0.35}$ alloys. We find the peaks to be well-explained by low energy $d-d$ excitations resulting from cobalt ions in two different low symmetry ligand fields. These are shown in Figure 3b. In model A the peaks are caused by surface metal ions ligated by an adsorbate. In model $\mathrm{B}$ the excitations stem from metal ions coordinated by ligands in a molecular complex. ${ }^{15}$ A third possibility would be that maximally the first atomic layer of the nanoparticles has interstitial oxygen in the cobalt lattice. In that case surface cobalt ions would possess a low symmetric ligand field, too. It is however unlikely that exactly this situation occurred in all three samples I-III that we protected from the atmosphere during all stages of sample handling. Moreover, even if we consider this situation, the cobalt ion symmetry would be similar as considered in model B with a maximum of four planar oxygen atoms around the surface cobalt ions. We therefore do not further discuss this model and elaborate below on models A and $\mathrm{B}$.

Figure 4 addresses model A. The observed absence of resonant X-ray Raman features on a pure metal, together with the low ligand coordination number of a ligated surface metal ion, and thus a weak ionic ligand field, raises the expectation that metal ions that have part of their electrons localized in a surface bond might reveal low energy $\mathrm{d}-\mathrm{d}$ excitations.

For model A the mode of the strongest carboxylate binding on a cobalt surface was first determined from configuration optimization DFT calculations. ${ }^{26}$ Figure $4 a$ shows the stable 


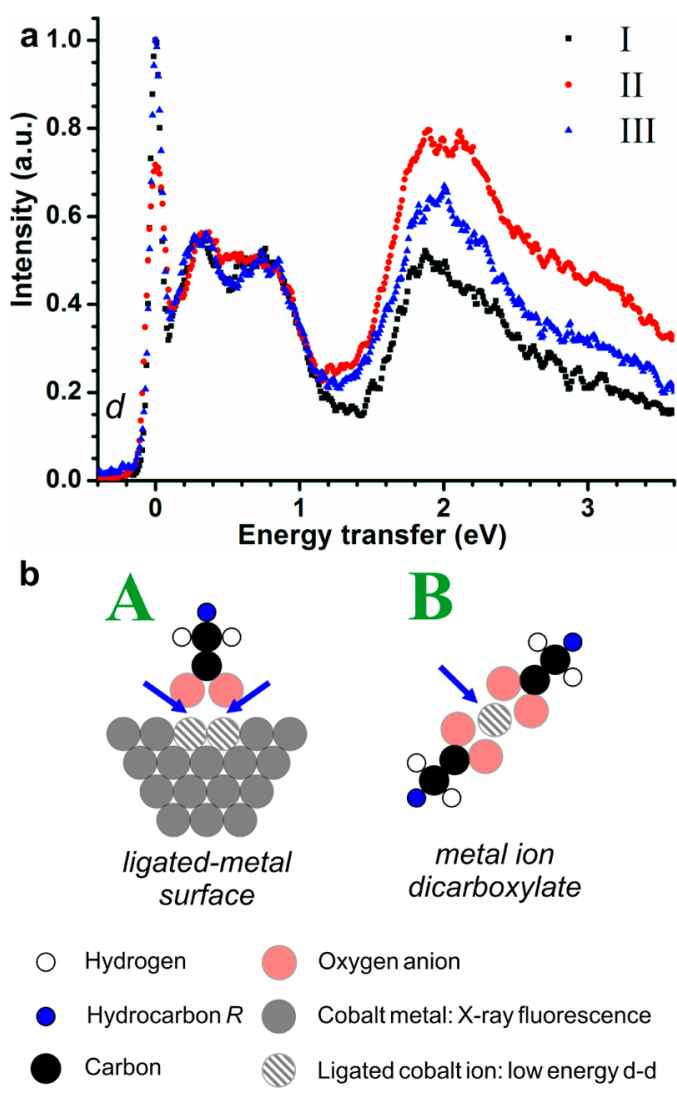

Figure 3. (a) Cobalt 2p3d RXES spectra of nanoparticle systems I-III at excitation energy $d$ all show resonant Raman peaks at 0.3 and 0.75 eV. (b) Schematic representation of two atomic environments which may give rise to these features: surface metal ions ligated by an adsorbate (model A) and a metal ion in a molecular complex (model B).

geometry of acetate, used to mimic oleate, bonded to a cobalt surface. It consists of two oxygen atoms on top of two cobalt atoms. The bonding mode is similar in both cobalt cluster acetate systems possessing a total charge of, respectively, +1 and -1 . Prior to geometry optimization it was determined with DFT that the bond dissociation energy of cobalt with oleate was higher than that with OA or TOPO (the synthesis surfactants). The calculations also revealed that cobalt(II)oleate bonds are favorable over cobalt(0)-oleate bonds. All these results are in line with experimental observations. ${ }^{4,47,48}$

Using the determined geometry, TD-DFT calculations were performed and the results are given in Figure $4 \mathrm{~b}$. A frequencydependent mean polarizability $\alpha(\omega)$ describes the reordering of electron states when a time-dependent electric field with frequency $\omega(t)$ is applied that mimics the photon radiation field. ${ }^{33}$ This allows the calculation of electron absorption transitions within a single particle framework. As such it can equally predict and explain low energy, single-electron RXES $\mathrm{d}-\mathrm{d}$ excitations. In order for TD-DFT to explain RXES instead of UV/vis spectra of multimetallic species, we used a single ion model of the relevant bond. A cobalt(II) methanolate species, with a fixed $\mathrm{Co}-\mathrm{O}-\mathrm{C}$ bond angle mimicking that of dicobalt carboxylate (fixed at $120^{\circ}$ vs $116^{\circ}$ ), gives TD-DFT transitions at $0.31,0.48,0.75$, and $0.80 \mathrm{eV}$. As a reference, the transitions of cobalt(II) trimethylphosphine oxide, used to mimic a possible cobalt(II) trioctylphosphine oxide bond, and of a cobalt(II) oxide bond are shown. These transitions do not match the experimental RXES peaks at 0.3 and $0.75 \mathrm{eV}$, which suggests that they are not significantly present in systems I-III. Effects of multiple metal ions, metal valence, and bond angle on TD-DFT results are given in the Supporting Information, Figure S2.

Subsequently Mulliken population analysis is used to determine the contribution of atomic orbitals to the molecular orbitals (MOs) in the bond. The TD-DFT transitions at 0.31

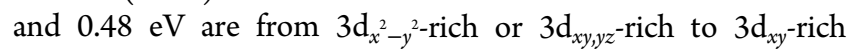
MOs, respectively. The transitions at 0.75 and $0.80 \mathrm{eV}$ are from $3 \mathrm{~d}_{x z, y z}$-rich to $3 \mathrm{~d}_{y z, x z}$-rich or $3 \mathrm{~d}_{z^{2}}$-rich MOs, respectively. The $3 \mathrm{~d}_{x^{2}-y^{2}}$-rich, $3 \mathrm{~d}_{x z, y z}$-rich, and $3 \mathrm{~d}_{x y}$-rich MOs consist of 98, 91, and $89 \%$ of their $3 \mathrm{~d}$ atomic orbitals, and they can be considered weak or nonbonding. The first two transitions occur between these states. The TD-DFT and Mulliken analyses now provide restrictions for the input values of the LFM calculations as shown in Figure 4c. LFM theory takes many-electron states into account that are not included in DFT/TD-DFT. The first restrictions are the origin and energies of the four low energy TD-DFT transitions as given in Table 2. The main atomic orbital character is shown of the MOs between which the transitions occur. As a second restriction, the energy difference between $3 \mathrm{~d}_{x^{2}-y^{2}}$ and $3 \mathrm{~d}_{x y}$ orbitals is $-10 \mathrm{Dq}$ in the first approximation, since LFM calculations are performed in tetragonal $D_{4 h}$ symmetry. ${ }^{10} 10 \mathrm{Dq}$ should thus be approximately $-0.3 \mathrm{eV}$. More details on the use of cobalt(II) methanolate as a mimic for the oleate nanoparticle bond and on the restrictions obtained from TD-DFT for LFM calculations can be found in the Supporting Information, Figure S3.

To determine the other ligand field parameters Ds and Dt, an orbital projection method (OPM) is used. ${ }^{45,46}$ These LFM calculations allow the description of many-electron states as fractions of single-particle states. As such, LFM parameters were identified that (1) yield states at energies of the TD-DFT transitions and (2) yield many-electron states that consist mainly of single-electron fractions that match the Mulliken determined atomic orbital character. To this end Ds and Dt are systematically varied independently in steps of $0.01 \mathrm{eV}$ around initial guess values obtained from simple one-electron rules in the appropriate symmetry $\left(\right.$ here $\left.D_{4 h}\right) .{ }^{10}$ The LFM parameters are $10 \mathrm{Dq}=-0.2 \mathrm{eV}$, Ds $=-0.05 \mathrm{eV}$, and $\mathrm{Dt}=-0.07 \mathrm{eV}$, as given in Table 1 . The energies of the resulting states and their main single particle fractions are given in Table 2 . The ground state is mainly a mixture of $e b_{2} a_{1}$ and $b_{2} a_{1} b_{1}$ electron hole states at 0 and $0.025 \mathrm{eV}$. An excited state at $0.26 \mathrm{eV}$ exists with mainly $\mathrm{ea}_{1} \mathrm{~b}_{1}$ hole state character. This yields transitions from $3 \mathrm{~d}_{x^{2}-y^{2}-}$ rich or $3 \mathrm{~d}_{x z, y z}$-rich to $3 \mathrm{~d}_{x y}$-rich states at 0.26 and $0.24 \mathrm{eV}$, respectively. At 0.51 and $0.71 \mathrm{eV}$ eeb $b_{2}$ and eeb $_{1}$ electron hole states exist, respectively. This yields a transition from $3 \mathrm{~d}_{x z, y z}$-rich

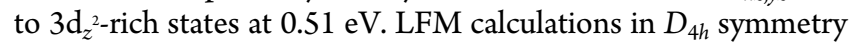
do not yield the third TD-DFT transition from $3 \mathrm{~d}_{x z, y z}$-rich to $3 \mathrm{~d}_{x z, y z}$-rich states, because $3 \mathrm{~d}_{x z}$ and $3 \mathrm{~d}_{y z}$ are in this symmetry formally degenerate. The LFM calculations do however reveal a double electron-double hole transition at $0.71 \mathrm{eV}$ : from $3 \mathrm{~d}_{x^{2}-y^{2}}$ $+3 \mathrm{~d}_{y z, x z}$ to $3 \mathrm{~d}_{x y}+3 \mathrm{~d}_{z^{2}}$. Overall, acceptable agreement between the energies of the TD-DFT and LFM transitions is found, providing a firm basis for the choice of the ligand field parameters used in model A. The resulting LFM cobalt $2 \mathrm{p} 3 \mathrm{~d}$ RXES calculation at excitation energy $d$ is shown in Figure $4 c$ under the experimental spectra acquired on the nanoparticles. The convoluted simulation accounts well for the peaks at 0.3 and $0.75 \mathrm{eV}$. 
A

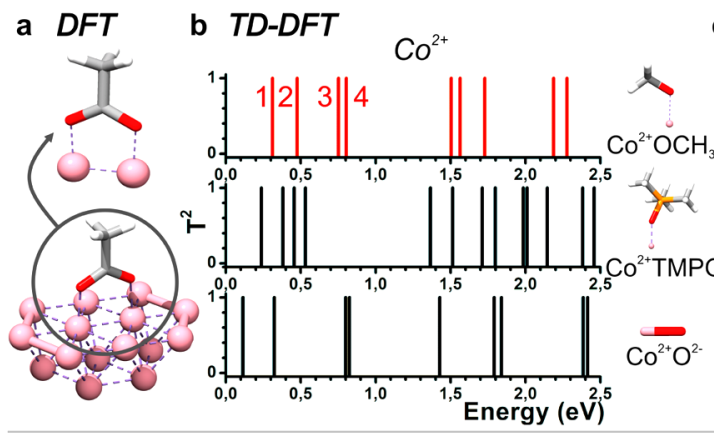

C $L F M$
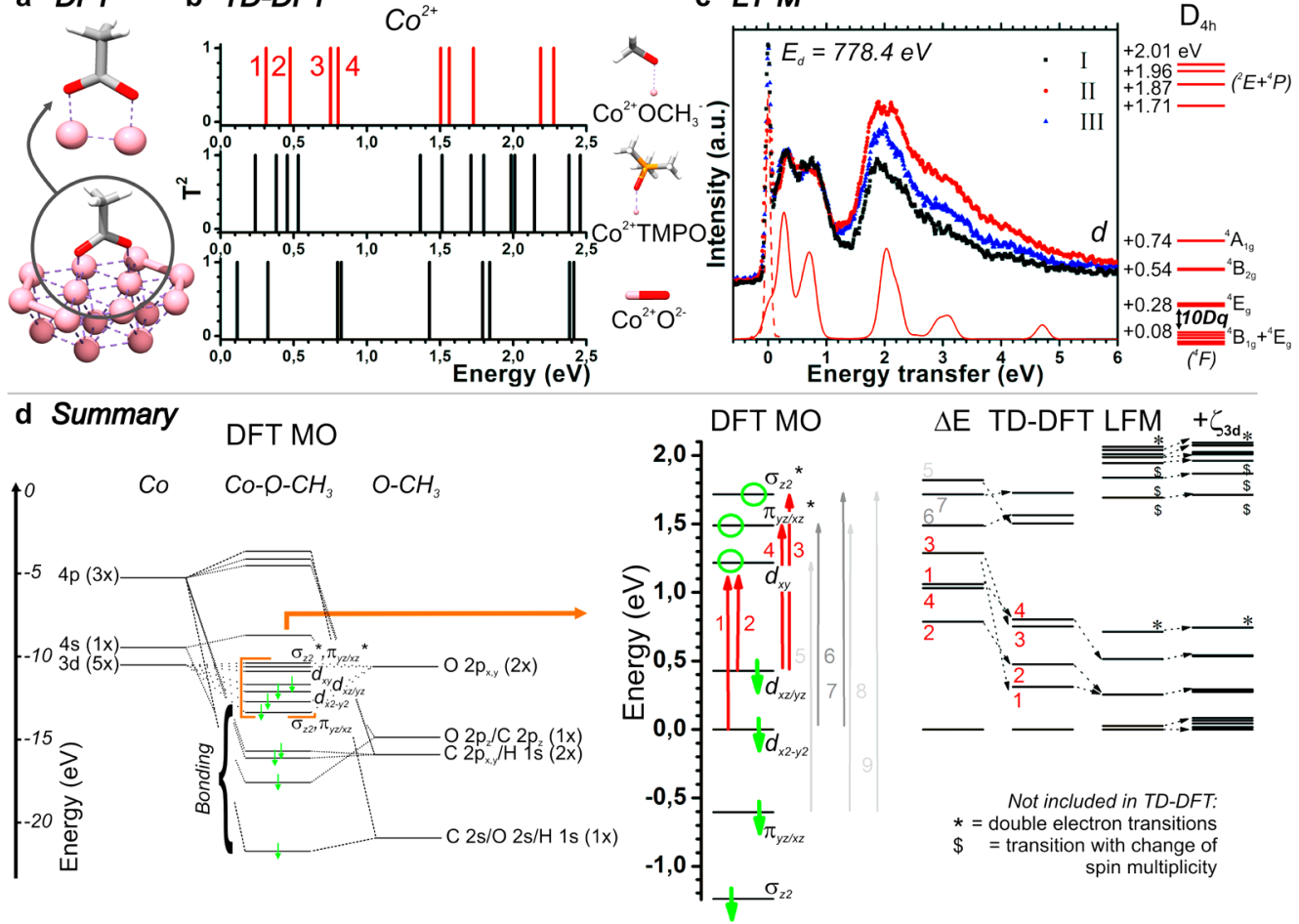

Figure 4. Electronic structure calculations used in model A. (a) The stable cobalt carboxylate geometry calculated by DFT. (b) Electron transitions between the MOs in the different cobalt(II)-ligand bonds as calculated by TD-DFT. (c) Experimental 2p3d cobalt RXES spectra (scatter plots) of nanoparticle systems I-III acquired at excitation energy $d(778.4 \mathrm{eV})$ together with the corresponding LFM RXES calculation (red line) of model A. The corresponding symmetry labeled states are shown to the right. The red dashed spectrum at $0 \mathrm{eV}$ was taken off the sample to reveal background X-ray scattering. (d) MO diagram of the bond (left) and the energies of the electron transitions as calculated by DFT, TD-DFT, and LFM (right).

Table 2. Mulliken and OPM Characters of the Transitions in Model A and Their Energetic Values (in eV) Found by TDDFT and LFM

\begin{tabular}{|c|c|c|c|c|}
\hline & $\begin{array}{l}\text { Mulliken char of } \\
\text { transitions }\end{array}$ & $\begin{array}{l}\text { TD-DFT } \\
\text { energy }\end{array}$ & $\begin{array}{l}\text { OPM char of } \\
\text { transitions }\end{array}$ & $\begin{array}{l}\text { LFM } \\
\text { energy }\end{array}$ \\
\hline 1 & $3 \mathrm{~d}_{x^{2}-y^{2}}$ to $3 \mathrm{~d}_{x y}$ & 0.31 & $3 \mathrm{~d}_{x^{2}-y^{2}}$ to $3 \mathrm{~d}_{x y}$ & 0.26 \\
\hline 2 & $3 \mathrm{~d}_{x z, y z}$ to $3 \mathrm{~d}_{x y}$ & 0.48 & $3 \mathrm{~d}_{x z, y z}$ to $3 \mathrm{~d}_{x y}$ & 0.24 \\
\hline 3 & $3 \mathrm{~d}_{x z, y z}$ to $3 \mathrm{~d}_{y z, x z}$ & 0.75 & $3 \mathrm{~d}_{x z, y z}$ to $3 \mathrm{~d}_{z^{2}}$ & 0.51 \\
\hline 4 & $3 \mathrm{~d}_{x z, y z}$ to $3 \mathrm{~d}_{z^{2}}$ & 0.80 & $\begin{array}{l}3 \mathrm{~d}_{x^{2}-y^{2}}+3 \mathrm{~d}_{x z, y z} \text { to } 3 \mathrm{~d}_{x y} \\
\quad+3 \mathrm{~d}_{z^{2}}\end{array}$ & 0.71 \\
\hline
\end{tabular}

${ }^{a}$ Main atomic orbital character of states is indicated.

Figure $4 \mathrm{~d}$ finally summarizes the model A calculations. The left panel shows a DFT MO diagram for the cobalt(II) methanolate bond. It shows the bonding and antibonding MOs between the cobalt $4 s$ and $4 p$ levels with ligand $2 s$ and $2 p$ orbitals. It also shows cobalt $3 \mathrm{~d}$ nonbonding states and (anti)bonding MOs with two of the ligand oxygen $2 p$ orbitals. The right panel in Figure $4 \mathrm{~d}$ gives the density functional energies in the first three columns and the multiplet energies in the last two columns. The first column shows the energies of the partly filled spin-down populated frontier orbitals, where the cobalt $3 \mathrm{~d}_{x^{2}-y^{2}}^{2}$-derived $\mathrm{MO}$ is set at $0 \mathrm{eV}$. The second column gives the energy differences between the occupied and empty $3 \mathrm{~d}$ orbitals. These energies would relate to $\mathrm{d}-\mathrm{d}$ transitions if one would assume that the other orbitals would not reorder in such a process. The TD-DFT results in the third column explicitly give the energies of the reordered MOs. This shifts the transitions to lower energies. The fourth column gives the LFM results. The consequence of the LFM multielectron character is that the fourth TD-DFT transition is shifted down from 0.80 to $0.51 \mathrm{eV}$ and that a new transition at $0.71 \mathrm{eV}$ occurs. This is a two electron-two hole excitation, or double excitation, and is indicated with an asterisk $(*)$. The final energy scheme is again the LFM calculation, but now with inclusion of the $3 \mathrm{~d}$ spin-orbit coupling, which was used for the RXES calculation shown in Figure 4c.

In model $\mathrm{B}$ the low energy $\mathrm{d}-\mathrm{d}$ excitations stem from metal ions coordinated by ligands in a molecular complex. This model is treated in Figure 5. Next to the 2p3d cobalt RXES spectrum of the nanoparticles, an experimental spectrum of a molecular cobalt(II) dioleate is shown. ${ }^{15}$ It is clear that this spectrum resembles the nanoparticle spectra. This leads to the alternative hypothesis that molecular species, dissolved from the nanoparticles, could in principle be the origin of the observed $\mathrm{d}-\mathrm{d}$ excitations at $\sim 0.3$ and $\sim 0.75 \mathrm{eV}$. We reported the LFM calculations for this cobalt(II) ion in the oleate in tetragonal $D_{4 h}$ symmetry. ${ }^{15}$ In short, an excitation at $0.29 \mathrm{eV}$ is found, caused by a ${ }^{4} \mathrm{E}_{\mathrm{g}}\left({ }^{4} \mathrm{~F}\right) \leftarrow{ }^{4} \mathrm{~A}_{2 \mathrm{~g}}\left({ }^{4} \mathrm{~F}\right)$ transition. ${ }^{53}$ A second transition at $0.79 \mathrm{eV}$ is found to be a ${ }^{4} \mathrm{E}_{\mathrm{g}}\left({ }^{4} \mathrm{~F}\right)+{ }^{4} \mathrm{~B}_{2 \mathrm{~g}}\left({ }^{4} \mathrm{~F}\right) \leftarrow$ ${ }^{4} \mathrm{~A}_{2 \mathrm{~g}}\left({ }^{4} \mathrm{~F}\right)$ transition.

\section{DISCUSSION}

In both models $\mathrm{A}$ and $\mathrm{B}$ the $2 \mathrm{p} 3 \mathrm{~d}$ RXES excitations at 0.3 and $0.75 \mathrm{eV}$ are interpreted to be low energy $\mathrm{d}-\mathrm{d}$ excitations due to a low symmetry ligand field. In model A this concerned a ligated surface metal ion; in model B this was a cobalt ion in a planar 4-fold oxygen ligation environment of a molecular 


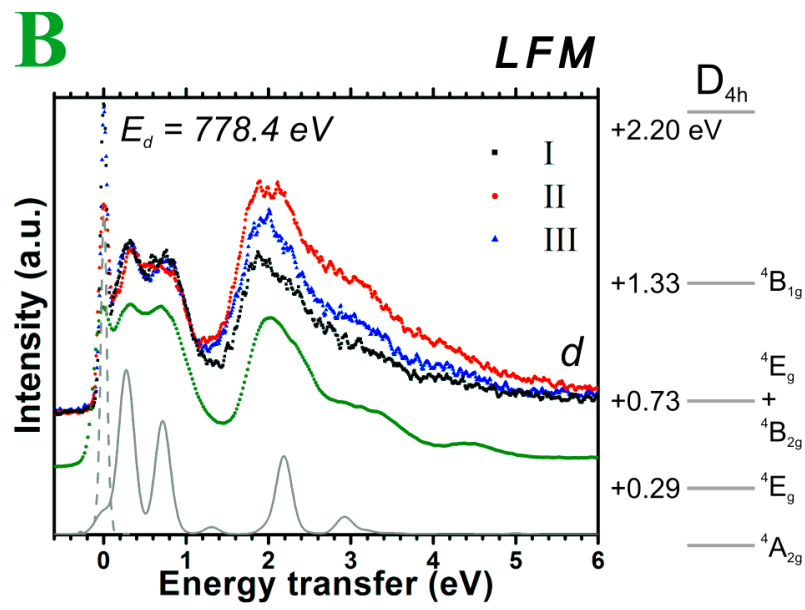

Figure 5. Experimental 2p3d cobalt RXES spectra of the cobalt bearing nanoparticles (black, red, blue) and of molecular cobalt oleate (green) acquired at excitation energy $d(778.4 \mathrm{eV}$; scatter plots). The corresponding LFM calculation of model B is also shown (gray line) with the symmetry labeled states to the right. The gray dashed spectrum at $0 \mathrm{eV}$ was taken off the sample to reveal background $\mathrm{X}$-ray scattering.

complex. An important conclusion is thus that the energies at which low symmetry $d-d$ excitations occur can be similar for different symmetries. The orbital character of the transitions in the different low symmetric environments is however different. We can use this to extend our discussion by considering the XAS spectra and the RXES spectra acquired at the other excitation energies than energy $d$, on nanoparticle systems IIII. We note here that one earlier study has suggested that $2 \mathrm{p} 3 \mathrm{~d}$ RXES could reveal ligand-adsorbate interactions on cobalt nanoparticles. ${ }^{54}$ This was however based on spectral features, including nanoparticle size dependent peaks, that we never observed and we therefore limit our discussion to our own experimental and theoretical data.

An often practiced method in XAS spectroscopic studies is to sum weighted spectra of different constituents that make up a spectrum. We checked whether such an approach could discriminate between models $\mathrm{A}$ and $\mathrm{B}$ as an explanation of the observed peaks. Figure 6 shows the experimental $2 p_{3 / 2}$ XAS spectra of the $3.6 \mathrm{~nm} \mathrm{fcc} \mathrm{Co}_{0.65} \mathrm{Ni}_{0.35}$ and the 5.0 and $8.4 \mathrm{~nm} \varepsilon$ Co nanoparticles. It also shows a reference cobalt metal XAS spectrum taken from Chen et al. ${ }^{55}$ The LFM calculated $2 \mathrm{p}_{3 / 2}$ XAS spectra obtained with the parameters of models $\mathrm{A}$ and $\mathrm{B}$ are displayed. We estimate (see Supporting Information) the atomic surface fraction to be $20 \%$ for the $\mathrm{Co}_{0.65} \mathrm{Ni}_{0.35}$ nanoparticles in case the alloy composition extends to the outer atomic shell, or maximally $40 \%$ if the outer atomic shell consists fully of cobalt. For the $\varepsilon$-Co 5.0 and $8.4 \mathrm{~nm}$ particles we estimate the surface fractions to be 15 and $10 \%$, respectively. We make now weighted sums of the metal spectra with 10,20 , 30 and $40 \%$ of the LFM spectra calculated in models A and B, to see which of the spectra fit the experimental spectra best. For model A this would thus represent a situation in which $10-40 \%$ of the cobalt ions are in the surface; for model B this would represent a situation where $10-40 \%$ of the cobalt ions are dissolved from the nanoparticles. Figure 6 shows that the weighted spectra of both models qualitatively match with experimental XAS spectra. We therefore consider such an approach to be inadequate to discriminate between the two models. Note in this context that an advantage of $2 \mathrm{p} 3 \mathrm{~d}$ RXES is

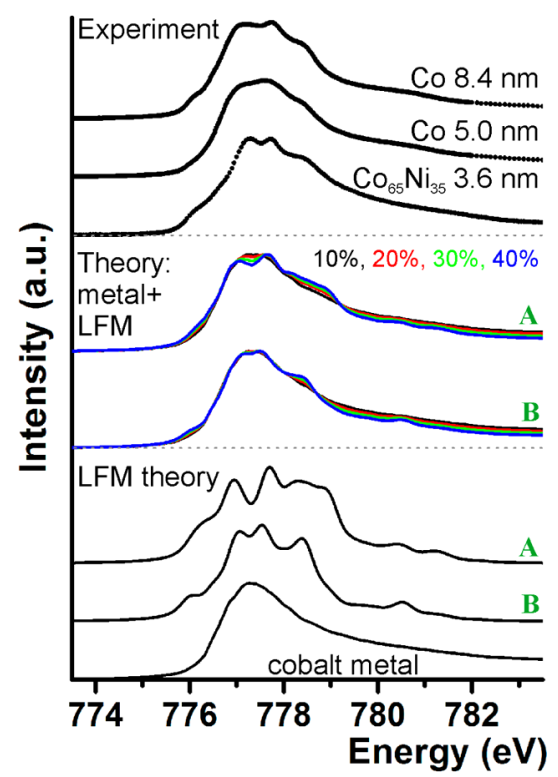

Figure 6. Experimental $2 \mathrm{p}_{3 / 2}$ XAS spectra of the studied nanoparticle systems (top). Reference cobalt metal spectrum together with LFM calculated $2 \mathrm{p}_{3 / 2}$ XAS spectra of models A and B (bottom). Weighted LFM and metal spectra (middle). The 10, 20, 30 and $40 \%$ refer to the fraction of the LFM spectrum in the weighted spectrum.

that weighing with a metallic signal is unnecessary for the resonant Raman part of the spectra, since formal metals in practice do not reveal such features.

The resonant behavior of the nanoparticles, the molecular cobalt oleate, and the theoretical models do however shed more light on the physical origin of the RXES peaks at 0.3 and 0.75 eV. Figure 7a shows the experimental spectra. The spectra are normalized to the $\mathrm{d}-\mathrm{d}$ transitions at 0.3 and $0.75 \mathrm{eV}$. The similarity between the nanoparticles and the molecular cobalt oleate data is clear. This suggests that next to the cobalt bearing nanoparticles some molecular cobalt oleate is likely present in the samples. Important differences between the experimental spectra however also exist. Most apparent is the additional intensity around $4-5 \mathrm{eV}$ in the data acquired at energy $e$ for the nanoparticles compared to the molecular species. This is normal X-ray fluorescence from formal cobalt metal in the nanoparticles. The dashed line in the spectra also indicates where it is expected to occur in the spectra measured at $a-d$ based on the incident X-ray energies. For these spectra additional fluorescence intensity is equally present, albeit relative to the $\mathrm{d}$-states less due to exciting on $\mathrm{L}_{3}$-edge resonance. Metal fluorescence also explains the increased intensity around $0.3 \mathrm{eV}$ in the spectra acquired at energy $a$. RXES cross sections for different $d$-states and metal fluorescence are unknown, and RXES spectra are experimentally difficult to quantify, because they depend on many variables, including the excitation energy. From the relative intensities of the $\mathrm{d}-\mathrm{d}$ and fluorescence features one can therefore not quantify the percentage of ionic versus metal cobalt, or the amount of molecular species versus nanoparticles. However, the nanoparticle systems were thoroughly washed before measurements and molecular coexisting species should be present in minimal amounts, most likely resulting from leaching. The counting times for the nickel RXES metal spectra were significantly longer than for the cobalt RXES spectra of the $\mathrm{Co}_{0.65} \mathrm{Ni}_{0.35}$ nanoparticles, indicating that cross sections for ionic species that generate $\mathrm{d}-\mathrm{d}$ excitations are considerably 


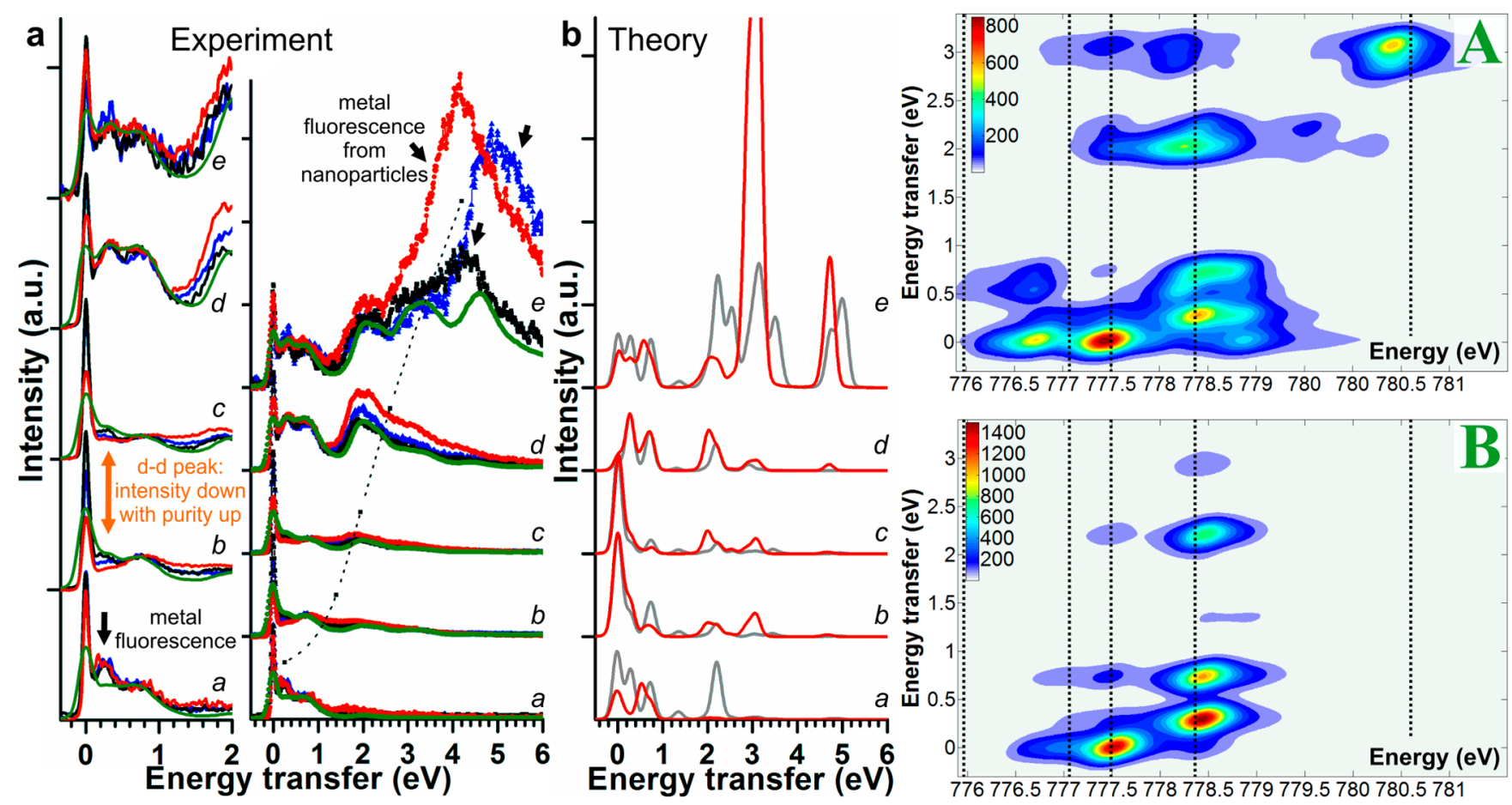

Figure 7. (a) Experimental 2p3d RXES spectra acquired at excitation energies $a-e$ for systems I-III (black, red, blue, respectively) and molecular cobalt(II) dioleate (green). The dashed line in the second panel indicates the energy at which normal fluorescence occurs. (b) LFM 2p3d RXES spectra (left) at excitation energies $a-e$ for models A (red) and B (gray) and LFM 2p3d RXES planes (right) in which the cross sections at energies $a-e$ are indicated by black dotted lines.

higher than for metal fluorescence, which implies that minimal amounts of coexisting molecular species are indeed likely to dominate the spectral intensity of a large amount of formal metal atoms present in the cores of the metal nanoparticles. In any case, we can say that the stronger the relative intensity of fluorescence versus $\mathrm{d}-\mathrm{d}$ excitations within one spectrum acquired at energy $e$, the larger the relative part of formal metal atoms in a nanoparticle system. Based on this, it is concluded that systems II and III contain less molecular oleate species and are purer nanoparticle systems than system I. In addition, based on particle size arguments, one would expect the $8.4 \mathrm{~nm}$ particles of system I to contain the most formal metal, followed by the 5.0 and $3.6 \mathrm{~nm}$ particles, respectively. This implies that the relative amount of molecular species is even higher in this system. Also, the spectra in system I match the experimental molecular cobalt oleate data best at all excitation energies. In addition, for the $5.0 \mathrm{~nm}$ cobalt nanoparticles of system II the XAS spectrum most resembles a white line as expected for a metal, which confirms that this is the purest nanoparticle system. In this respect it is interesting that, at $0.3 \mathrm{eV}$ in the spectra acquired at excitation energies $b$ and $c$, the spectral intensity for systems II and III is low compared to the peak at $0.75 \mathrm{eV}$. This shows that a second, nonmetallic cobalt species is present that generates a different $\mathrm{d}-\mathrm{d}$ profile from the molecular reference.

The complete calculated LFM RXES planes for all excitation energies over the cobalt $2 \mathrm{p}_{3 / 2}$ XAS edge for both models $A$ and $B$ are given on the right side of Figure $7 \mathrm{~b}$. Note that LFM calculations do not calculate the elastic peak and normal fluorescence and therefore fail to calculate additional intensities from the formal metal parts of the nanoparticles. From these planes the resemblance between the models is clear, as well as their differences, especially around 776.8 and $780.3 \mathrm{eV}$. Cross sections of the planes at the excitation energies $a-e$ yield the theoretical 2p3d RXES spectra as displayed adjacent to the experimental spectra. The comparison with experiment shows that the surface species model A fails for energies $a$ and $e$, as one peak is predicted around $0.6 \mathrm{eV}$ at energy $a$ and a very strong peak is predicted around $3 \mathrm{eV}$ for energy e. Model $\mathrm{B}$ does a better job in reproducing the spectra and only fails when predicting an intense peak around $2.1 \mathrm{eV}$ at energy $a$. This confirms again that much of the experimental RXES spectra can be explained by a model $\mathrm{B}$ type cobalt ion coordination environment, probably mainly due to the presence of leached molecular cobalt oleate. However, especially for system II, which is the purest nanoparticle sample, model B cannot explain the drop in intensity at energies $b$ and $c$ for the excitation at $0.3 \mathrm{eV}$ in the experimental spectra. Model A also cannot explain this trend, despite our novel approach to correlate TD-DFT to LFM calculations in order to limit the number of possible LFM interpretations. ${ }^{56-63}$ In this context it should be mentioned that comparing RXES spectra at one excitation energy, as shown here for the spectra at energy $d$, may be used to effectively discriminate high and low symmetric ion coordination environments from each other, ${ }^{15}$ but that it requires two-dimensional RXES planes, or at least a few spectra acquired at multiple energies, to discriminate different low symmetric coordination environments. Full two-dimensional (2D) RXES planes possess thus a large fingerprinting capacity for characterization. We further note that in future measurements clean surface science samples with a single molecular adsorbate layer may be used to conclude whether the $d-d$ excitation profile observed on $5.0 \mathrm{~nm}$ particles is indeed from ligated cobalt surface species, in which case 2 p3d RXES would be able to uniquely measure the bond between a metal surface species and the binding atom of an adsorbate. Here we chose 
however first to study nanoparticles because (1) they possess a relatively high surface to bulk ratio and thus many surface species and (2) the X-ray probe is negligibly affected by saturation and self-absorption effects since the particles are optically thin.

\section{CONCLUSIONS}

We reported an extensive set of high resolution ( $\mathrm{fwhm}<0.1$ $\mathrm{eV}$ ) cobalt and nickel 2p3d RXES spectra on $8.4 \mathrm{~nm} \varepsilon$-Co, 5.0 $\mathrm{nm} \varepsilon$-Co, and $3.6 \mathrm{~nm} \mathrm{Co}_{0.65} \mathrm{Ni}_{0.35}$ nanoparticles. All batches show peaks of previously unknown origin at 0.3 and $0.75 \mathrm{eV}$ in the cobalt RXES spectra. The nickel RXES spectra of the alloy particles show only X-ray fluorescence, indicating that it is fully metallic. The low energy cobalt RXES peaks are interpreted to be low energy cobalt $\mathrm{d}-\mathrm{d}$ excitations due to cobalt ions in a low symmetry ligand field. Two models were investigated that could possibly explain the peaks. In model $\mathrm{A}$, a novel correlation between TD-DFT and LFM theory was used to show that the peaks could stem from surface metal ions coordinated by carboxylate ligands. In model $\mathrm{B}$, the peaks originate from a cobalt molecular species that might coexist with the nanoparticles. Using the three different nanoparticle batches and 2D LFM RXES planes, it is concluded that model A fails to explain the spectra and that varying minor degrees of molecular cobalt oleate are indeed present in the wet chemically prepared nanoparticle systems. This is surprising as the nanoparticle systems were thoroughly washed prior to measurements, but it shows that minority species of, probably leached, metal ions dominate the 2p3d RXES spectra of further formally metallic systems. It is also shown however that a decrease in the relative amount of molecular species reveals a different $d-d$ intensity profile in the purest nanoparticle system (II), unrelated to molecular cobalt oleate and possibly related to surface-ligated cobalt ions. Albeit the chemical heterogeneity of the systems, 2p3d RXES revealed the particles to be metallic, to coexist with varying minor degrees of molecular species, and that a second cobalt species is present. The 2p3d RXES should equally allow the study of applied complex chemical systems as present in, for example, heterogeneous catalysts and batteries.

\section{ASSOCIATED CONTENT}

\section{S Supporting Information}

Detailed experimental and computational procedures and supplementary data and discussions. ${ }^{64,65}$ This material is available free of charge via the Internet at http://pubs.acs.org.

\section{AUTHOR INFORMATION}

\section{Corresponding Author}

*E-mail: matti.van-schooneveld@cec.mpg.de (M.M.S.); f.m.f. degroot@uu.nl(F.M.F.G.).

\section{Notes}

The authors declare no competing financial interest.

\section{ACKNOWLEDGMENTS}

We thank Karen Hemelsoet for discussions. This work was financially supported by a VICI grant (FMFdG) of The Netherlands Organization for Scientific Research (NWO-CW).

\section{REFERENCES}

(1) Rosei, F.; Schunack, M.; Naitoh, Y.; Jiang, P.; Gourdon, A.; Laegsgaard, E.; Stensgaard, I.; Joachim, C.; Besenbacher, F. Properties of Large Organic Molecules on Metal Surfaces. Prog. Surf. Sci. 2003, $71,95-146$.

(2) Cramer, C. J.; Truhlar, D. G. Density Functional Theory for Transition Metals and Transition Metal Chemistry. Phys. Chem. Chem. Phys. 2009, 11, 10757-10816.

(3) Nilsson, A.; Pettersson, L. G. M.; Hammer, B.; Bligaard, T.; Christensen, C. H.; Nørskov, J. K. The Electronic Structure Effect in Heterogeneous Catalysis. Catal. Lett. 2005, 100, 111-114.

(4) Nilsson, A.; Pettersson, L. G. M. Chemical Bonding on Surfaces Probed by X-ray Emission Spectroscopy and Density Functional Theory. Surf. Sci. Rep. 2004, 55, 49-167.

(5) Stöhr, J. NEXAFS Spectroscopy, 1st ed.; Springer-Verlag: New York, 1992; Vol. 25.

(6) Ertl, G. Reactions at Surfaces: From Atoms to Complexity (Nobel Lecture). Angew. Chem., Int. Ed. 2008, 47, 3524-3535.

(7) Nilsson, A.; Pettersson, L. G. M.; Nørskov, J. K. Chemical Bonding at Surfaces and Interfaces, 1st ed.; Elsevier: Amsterdam, 2008.

(8) Gel'mukhanov, F.; Ågren, H. Resonant Inelastic X-Ray Scattering with Symmetry-Selective Excitation. Phys. Rev. A 1994, 49, 43784389.

(9) Kotani, A. Theory of Resonant X-ray Emission Spectra in Strongly Correlated Electron Systems. J. Electron Spectrosc. Relat. Phenom. 2000, 110-111, 197-212.

(10) de Groot, F. M. F.; Kotani, A. Core Level Spectroscopy of Solids; CRC Press: Boca Raton, FL, 2008.

(11) Haverkort, M. W. Theory of Resonant Inelastic X-Ray Scattering by Collective Magnetic Excitations. Phys. Rev. Lett. 2010, $105,167404$.

(12) Ament, L. J. P.; van Veenendaal, M.; Devereaux, T. P.; Hill, J. P.; van den Brink, J. Resonant Inelastic X-Ray Scattering Studies of Elementary Excitations. Rev. Mod. Phys. 2011, 83, 705-767.

(13) Ghiringhelli, G.; Piazzalunga, A.; Dallera, C.; Trezzi, G.; Braicovich, L.; Schmitt, T.; Strocov, V. N.; Betemps, R.; Patthey, L.; Wang, X.; et al. SAXES, a High Resolution Spectrometer for Resonant $\mathrm{X}$-Ray Emission in the 400-1600eV Energy Range. Rev. Sci. Instrum. 2006, 77, 113108.

(14) Strocov, V. N.; Schmitt, T.; Flechsig, U.; Schmidt, T.; Imhof, A.; Chen, Q.; Raabe, J.; Betemps, R.; Zimoch, D.; Krempasky, J.; et al. High-Resolution Soft X-ray Beamline ADRESS at the Swiss Light Source for Resonant Inelastic X-ray Scattering and Angle-Resolved Photoelectron Spectroscopies. J. Synchrotron Radiat. 2010, 17, 631643.

(15) van Schooneveld, M. M.; Gosselink, R. W.; Eggenhuisen, T. M.; Al Samarai, M.; Monney, C.; Zhou, K. J.; Schmitt, T.; de Groot, F. M. F. A Multispectroscopic Study of 3d Orbitals in Cobalt Carboxylates: The High Sensitivity of 2p3d Resonant X-ray Emission Spectroscopy to the Ligand Field. Angew. Chem., Int. Ed. 2013, 52, 1170-1174.

(16) Henke, B. L.; Gullikson, E. M.; Davis, J. C. X-Ray Interactions: Photoabsorption, Scattering, Transmission, and Reflection at E $=50$ 30,000 eV, Z = 1-92. At. Data Nucl. Data Tables 1993, 54, 181-342.

(17) See Supporting Information for footnote.

(18) van Schooneveld, M. M.; Suljoti, E.; Campos-Cuerva, C.; Gosselink, R. W.; van der Eerden, A. M. J.; Schlappa, J.; Zhou, K.; Monney, C.; Schmitt, T.; de Groot, F. M. F. Transition-Metal Nanoparticle Oxidation in a Chemically Nonhomogenous Environment Revealed by 2p3d Resonant X-ray Emission. J. Phys. Chem. Lett. 2013, 4, 1161-1166.

(19) Puntes, V. F.; Krishnan, K. M.; Alivisatos, A. P. Colloidal Nanocrystal Shape and Size Control: The Case of Cobalt. Science 2001, 291, 2115-2117.

(20) van Schooneveld, M. M.; Campos-Cuerva, C.; Pet, J.; Meeldijk, J. D.; van Rijssel, J.; Meijerink, A.; Erné, B. H.; de Groot, F. M. F. Composition Tunable Cobalt-Nickel and Cobalt-Iron Alloy Nanoparticles Below $10 \mathrm{~nm}$ Synthesized Using Acetonated Cobalt Carbonyl. J. Nanopart. Res. 2012, 14, 1-13.

(21) Thornton, J. M. C.; Williams, R. H. An S/XPS Study of Hydrogen Terminated, Ordered Silicon (100) and (111) Surfaces Prepared by Chemical Etching. Phys. Scr. 1990, 41, 1047-1052. 
(22) Dumas, P.; Chabal, Y. J.; Jakob, P. Morphology of HydrogenTerminated $\mathrm{Si}(111)$ and $\mathrm{Si}(100)$ Surfaces Upon Etching in $\mathrm{HF}$ and Buffered-HF Solutions. Surf. Sci. 1992, 269, 867-878.

(23) Ghiringhelli, G.; Brookes, N. B.; Annese, E.; Berger, H.; Dallera, C.; Grioni, M.; Perfetti, L.; Tagliaferri, A.; Braicovich, L. Low Energy Electronic Excitations in the Layered Cuprates Studied by Copper L3 Resonant Inelastic X-Ray Scattering. Phys. Rev. Lett. 2004, 92, 117406.

(24) van Schooneveld, M. M.; Kurian, R.; Juhin, A.; Zhou, K.; Schlappa, J.; Strocov, V. N.; Schmitt, T.; de Groot, F. M. F. Electronic Structure of $\mathrm{CoO}$ Nanocrystals and a Single Crystal Probed by Resonant X-ray Emission Spectroscopy. J. Phys. Chem. C 2012, 116, $15218-15230$

(25) Neese, F.; Petrenko, T.; Ganyushin, D.; Olbrich, G. Advanced Aspects of Ab Initio Theoretical Optical Spectroscopy of Transition Metal Complexes: Multiplets, Spin-Orbit Coupling and Resonance Raman Intensities. Coord. Chem. Rev. 2007, 251, 288-327.

(26) Neese, F. The ORCA Program System. Wiley Interdiscip. Rev.: Comput. Mol. Sci. 2012, 2, 73-78.

(27) Becke, A. D. Density-Functional Exchange-Energy Approximation with Correct Asymptotic Behavior. Phys. Rev. A 1988, 38, 3098-3100.

(28) Perdew, J. P. Density-Functional Approximation for the Correlation Energy of the Inhomogeneous Electron Gas. Phys. Rev. B 1986, 33, 8822-8824.

(29) Schäfer, A.; Horn, H.; Ahlrichs, R. Fully Optimized Contracted Gaussian Basis Sets for Atoms Li to Kr. J. Chem. Phys. 1992, 97, 25712577.

(30) Schäfer, A.; Huber, C.; Ahlrichs, R. Fully Optimized Contracted Gaussian Basis Sets of Triple Zeta Valence Quality for Atoms Li to Kr. J. Chem. Phys. 1994, 100, 5829-5835.

(31) Weigend, F.; Ahlrichs, R. Balanced Basis Sets of Split Valence, Triple Zeta Valence and Quadruple Zeta Valence Quality for H to Rn: Design and Assessment of Accuracy. Phys. Chem. Chem. Phys. 2005, 7, 3297-3305

(32) Neese, F. A Critical Evaluation of DFT, Including TimeDependent DFT, Applied to Bioinorganic Chemistry. J. Biol. Inorg. Chem. 2006, 11, 702-711.

(33) Runge, E.; Gross, E. K. U. Density-Functional Theory for TimeDependent Systems. Phys. Rev. Lett. 1984, 52, 997-1000.

(34) Petrenko, T.; Neese, F. Analysis and Prediction of Absorption Band Shapes, Fluorescence Band Shapes, Resonance Raman Intensities, and Excitation Profiles Using the Time-Dependent Theory of Electronic Spectroscopy. J. Chem. Phys. 2007, 127, 164319.

(35) Bauernschmitt, R.; Ahlrichs, R. Treatment of Electronic Excitations within the Adiabatic Approximation of Time Dependent Density Functional Theory. Chem. Phys. Lett. 1996, 256, 454-464.

(36) Casida, M. E.; Jamorski, C.; Casida, K. C.; Salahub, D. R. Molecular Excitation Energies to High-Lying Bound States from TimeDependent Density-Functional Response Theory: Characterization and Correction of the Time-Dependent Local Density Approximation Ionization Threshold. J. Chem. Phys. 1998, 108, 4439-4449.

(37) Griffith, J. S. The Theory of Transition Metal Ions; Cambridge University Press: Cambridge, U.K., 1964.

(38) Stavitski, E.; de Groot, F. M. F. The CTM4XAS Program for EELS and XAS Spectral Shape Analysis of Transition Metal L Edges. Micron 2010, 41, 687-694.

(39) Cowan, R. D. Theoretical Calculation of Atomic Spectra Using Digital Computers. J. Opt. Soc. Am. 1968, 58, 808-818.

(40) Cowan, R. D. The Theory of Atomic Structure and Spectra; University of California Press: Berkeley, 1981.

(41) Thole, B. T.; van der Laan, G.; Fuggle, J. C.; Sawatzky, G. A.; Karnatak, R. C.; Esteva, J. M. 3d X-Ray-Absorption Lines and the $3 \mathrm{~d}^{9} 4 \mathrm{f}^{\mathrm{n}+1}$ Multiplets of the Lanthanides. Phys. Rev. B 1985, 32, 51075118.

(42) Kramers, H.; Heisenberg, W. Über die Streuung von Strahlung durch Atome. Z. Phys. 1925, 31, 681-708.

(43) Glatzel, P.; Bergmann, U. High Resolution 1s Core Hole X-Ray Spectroscopy in 3d Transition Metal Complexes-Electronic and Structural Information. Coord. Chem. Rev. 2005, 249, 65-95.
(44) Jørgensen, C. K. Modern Aspects of Ligand Field Theory; NorthHolland: Amsterdam, 1971.

(45) Wasinger, E. C.; de Groot, F. M. F.; Hedman, B.; Hodgson, K. O.; Solomon, E. I. L-edge X-ray Absorption Spectroscopy of NonHeme Iron Sites: Experimental Determination of Differential Orbital Covalency. J. Am. Chem. Soc. 2003, 125, 12894-12906.

(46) Piamonteze, C.; de Groot, F. M. F.; Tolentino, H. C. N.; Ramos, A. Y.; Massa, N. E.; Alonso, J. A.; Martínez-Lope, M. J. Spin-OrbitInduced Mixed-Spin Ground State in $\mathrm{RNiO}_{3}$ Perovskites Probed by XRay Absorption Spectroscopy: Insight Into the Metal-To-Insulator Transition. Phys. Rev. B 2005, 71, 020406.

(47) Lagunas, A.; Jimeno, C.; Font, D.; Solà, L.; Pericàs, M. A. Mechanistic Studies on the Conversion of Dicobalt Octacarbonyl into Colloidal Cobalt Nanoparticles. Langmuir 2006, 22, 3823-3829.

(48) Wu, N.; Fu, L.; Su, M.; Aslam, M.; Wong, K. C.; Dravid, V. P. Interaction of Fatty Acid Monolayers with Cobalt Nanoparticles. Nano Lett. 2004, 4, 383-386.

(49) de Groot, F. M. F.; Abbate, M.; van Elp, J.; Sawatzky, G. A.; Ma, Y. J.; Chen, C. T.; Sette, F. Oxygen 1s and Cobalt 2p X-Ray Absorption of Cobalt Oxides. J. Phys.: Condens. Matter 1993, 5, 22772288.

(50) See Supporting Information for footnote.

(51) See Supporting Information for footnote.

(52) See Supporting Information for footnote.

(53) See Supporting Information for footnote.

(54) Liu, H.; Guo, J.; Yin, Y.; Augustsson, A.; Dong, C.; Nordgren, J.; Chang, C.; Alivisatos, P.; Thornton, G.; Ogletree, D. F.; et al. Electronic Structure of Cobalt Nanocrystals Suspended in Liquid. Nano Lett. 2007, 7, 1919-1922.

(55) Chen, C. T.; Idzerda, Y. U.; Lin, H. J.; Smith, N. V.; Meigs, G.; Chaban, E.; Ho, G. H.; Pellegrin, E.; Sette, F. Experimental Confirmation of the X-Ray Magnetic Circular Dichroism Sum Rules for Iron and Cobalt. Phys. Rev. Lett. 1995, 75, 152-155.

(56) See Supporting Information for footnote.

(57) Anthon, C.; Bendix, J.; Schäffer, C. E. An Average-ofConfiguration Method for Using Kohn-Sham Density Functional Theory in Modeling Ligand-Field Theory. Inorg. Chem. 2003, 42, 4088-4097.

(58) Schäffer, C. E.; Anthon, C.; Bendix, J. Kohn-Sham DFT Results Projected on Ligand-Field Models: Using DFT to Supplement LigandField Descriptions and to Supply Ligand-Field Parameters. Coord. Chem. Rev. 2009, 253, 575-593.

(59) Uldry, A.; Vernay, F.; Delley, B. Systematic Computation of Crystal-Field Multiplets for X-Ray Core Spectroscopies. Phys. Rev. B 2012, 85, 125133.

(60) Bunău, O.; Arrio, M. A.; Sainctavit, P.; Paulatto, L.; Calandra, M.; Juhin, A.; Marvaud, V.; Cartier dit Moulin, C. Understanding the Photomagnetic Behavior in Copper Octacyanomolybdates. J. Phys. Chem. A 2012, 116, 8678-8683.

(61) Ikeno, H.; Tanaka, I.; Koyama, Y.; Mizoguchi, T.; Ogasawara, K. First-Principles Multielectron Calculations of $\mathrm{Ni} \mathrm{L}_{2,3} \mathrm{NEXAFS}$ and ELNES for $\mathrm{LiNiO}_{2}$ and Related Compounds. Phys. Rev. B 2005, 72, 075123.

(62) Ikeno, H.; Mizoguchi, T.; Tanaka, I. Ab Initio Charge Transfer Multiplet Calculations on the $\mathrm{L}_{2,3}$ XANES and ELNES of $3 \mathrm{~d}$ Transition Metal Oxides. Phys. Rev. B 2011, 83, 155107.

(63) Haverkort, M. W.; Zwierzycki, M.; Andersen, O. K. Multiplet Ligand-Field Theory Using Wannier Orbitals. Phys. Rev. B 2012, 85, 165113.

(64) Dinega, D. P.; Bawendi, M. G. A Solution-Phase Chemical Approach to a New Crystal Structure of Cobalt. Angew. Chem., Int. Ed. 1999, 38, 1788-1791.

(65) Respaud, M.; Broto, J. M.; Rakoto, H.; Fert, A. R.; Thomas, L.; Barbara, B.; Verelst, M.; Snoeck, E.; Lecante, P.; Mosset, A.; et al. Surface Effects on the Magnetic Properties of Ultrafine Cobalt Particles. Phys. Rev. B 1998, 57, 2925-2935. 